\title{
Dynamics of Spacecraft Plume/Magnetosphere Interactions in Geostationary Earth Orbit
}

\author{
Kelly A. Stephani* \\ Department of Mechanical Science and Engineering, University of Illinois at Urbana-Champaign \\ Iain D. Boyd ${ }^{\dagger}$ \\ Department of Aerospace Engineering, University of Michigan
}

\begin{abstract}
Detailed direct simulation Monte Carlo/Particle in Cell simulations involving the interaction of a hydrazine chemical rocket plume with the rarefied ambient magnetosphere are presented for steady thruster firings in geostationary Earth orbit (GEO). The hydrazine chemical rocket plume is modeled as a neutral gas mixture with thruster exit conditions corresponding to a mass flow rate of $5.0 \times 10^{-4} \mathrm{~kg} / \mathrm{s}$. The interaction of the steady plume with the rarefied magnetosphere is modeled using a combined direct simulation Monte Carlo/Particle in Cell methodology, allowing for a detailed assessment of non-equilibrium collisional and plasma-related phenomena relevant for these conditions. Charge exchange interactions between the plume chemical mixture and the ambient ions are modeled using detailed total and differential cross sections. The influence of the Earth's magnetic field on the development of the ion plume is also modeled assuming a field strength of $B=1.1 \times 10^{-7}$ T. The magnetic field is found to have only a minor impact on the development of the resulting neutral and ion plumes. The relative motion of the magnetic field lines with respect to the spacecraft is assumed to be negligible for a satellite in geosynchronous orbit, so spacecraft ions formed through charge exchange become trapped in gyroscopic orbit about field lines that are stationary relative to the spacecraft. The incorporation of the plume mixture into the model captures the variation in plume dissipation times per species, with the longest dissipation time corresponding to the $\mathrm{H}_{2}$ plume of 33 seconds.
\end{abstract}

\section{Introduction}

A fundamental understanding of plume dynamics is imperative for developing both predictive and mitigatory capabilities to avoid plume impingement on critical spacecraft surfaces. Chemical interactions between post-combustion neutral species generated by spacecraft thrusters and ambient ions in the upper atmosphere play an important role in determining the dynamic behavior of these plumes. In particular, the high-density neutral plume emitted during a thruster burn is subject to charge exchange reactions with the ambient ions. This interaction can alter the local magnetospheric properties, and lead to excitation of plasma waves. Studies of such interactions, both experimental and computational, have been centralized around LEO transportation spacecraft, including Space Shuttle, Soyuz, Progress and the Mir space station. ${ }^{1-6}$

The study by Burke et al. ${ }^{1}$ examined the energy distribution of positive, single-charge ions detected by the Shuttle Potential and Return Electron Experiment (SPREE) during a thruster burn of the Tethered Satellite System (TSS 1) mission. Data collected by this sensor included information regarding both energy and angular distributions of ions impacting the sensor, over ion energies ranging from 10 to $100 \mathrm{eV}$. This study also compared SPREE data with results from a two-dimensional collisionless molecular model. The model tracked trajectories of neutrals and pick-up ions (plume-related ions formed through charge transfer of plume neutrals with ambient ions) during a thruster burn event, and provided information regarding the distribution of ions that eventually impact the SPREE sensor. This allowed for a comparison between the

\footnotetext{
*Assistant Professor, Department of Mechanical Science and Engineering, 1206 W. Green St., Urbana, IL, Senior Member AIAA.

${ }^{\dagger}$ James E. Knott Professor, Department of Aerospace Engineering, 1320 Beal Ave, Ann Arbor, MI, Fellow AIAA.
} 
measured and predicted ion energy distributions. It was also found that significant scattering occurs near the thruster exit as well as after charge exchange between the neutral gas and ambient oxygen ions.

The present study aims to examine the interaction between spacecraft thruster plumes and the ambient magnetosphere in geostationary Earth orbit (GEO) conditions. While previous work has examined plume/ ionosphere interactions in low Earth orbit (LEO), ${ }^{7,8}$ this study focuses on non-equilibrium collisional and plasma-related phenomena at GEO, which is characterized by very rarefied and high-energy ambient conditions relative to LEO. The rarefied nature of the magnetosphere, as well as the surrounding plasma, requires the use of a combined direct simulation Monte Carlo/Particle in Cell (DSMC/PIC) methodology, which is detailed next. Results are presented for a steady spacecraft thruster burn, in which the neutral rocket plume is modeled as a mixture of three primary propellant species. The effects from the geomagnetic and self-consistent electric fields on the development and propagation of the neutral and ion plumes is also investigated.

\section{Modeling of Plume/Magnetosphere Interactions}

\section{A. DSMC/PIC Framework}

The charge exchange collisions between ambient ions and rocket plume combustion species occur under very low density conditions. The most appropriate numerical method for simulation of these phenomena is the direct simulation Monte Carlo (DSMC) method. ${ }^{9}$ The plasma formed in this process is subject to self-consistent electro-static fields, which is most appropriately modeled using the Particle in Cell (PIC) method. ${ }^{10}$ The combination of rarefied collisional and plasma phenomena relevant to the physical system of interest is therefore analyzed using MPIC, ${ }^{11}$ which uses the DSMC and PIC methods simultaneously to model the flow field.

\section{B. MEX/CEX Collision Dynamics}

The ambient magnetosphere model used in this study is comprised of $\mathrm{H}^{+}$, the primary ion species found at GEO. The spacecraft thruster ejects a high-density plume of neutral particles which expands into the surrounding ambient flow. Interaction of the chemical rocket plume mixture with the ambient $\mathrm{H}^{+}$leads to the formation of an ion plume mixture through charge exchange (CEX) reactions. The chemical system under consideration is thus comprised of seven chemical species: the ambient $\mathrm{H}^{+}$ions, and the hydrazine combustion products, $\mathrm{H}_{2}, \mathrm{~N}_{2}$ and $\mathrm{NH}_{3}$ and their corresponding ions, $\mathrm{H}_{2}^{+}, \mathrm{N}_{2}^{+}$and $\mathrm{NH}_{3}^{+}$.

The ambient $\mathrm{H}^{+}$ions are allowed to participate in both momentum exchange (MEX) and CEX interactions with the plume constituents, but the post-collision properties of $\mathrm{H}^{+}$are not updated in order to preserve the properties of the ambient environment. All of the chemical species comprising the spacecraft plume mixture participate in MEX/CEX interactions, and binary collisions and post-collision scattering are computed based on detailed total and differential cross sections. The rotational, vibrational and electronic internal structure of plume constituents is currently neglected in this work, but should be included to handle the energy transfer in non-resonant charge exchange collisions.

The collision details involving plasma mixtures requires a careful treatment for collisions between neutral and charged particles, particularly in cases where the collision partners are not like species. Within the DSMC method, collision dynamics are imposed in the center-of-mass frame of reference of the colliding particles. Collisions involving two neutral particles are processed using the Variable Hard Sphere (VHS) model, in which the probability of a collision is computed according to:

$$
P=\frac{\sigma g}{(\sigma g)_{\max }},
$$

where $\sigma$ is the total cross section and $g$ is the relative collision speed, and a candidate pair is selected for collision if $P>\mathcal{R}_{u}$, where $\mathcal{R}_{u}$ is a uniformly distributed random number. Heavy particle interactions are treated according to standard DSMC collision dynamics, with the possibility of a charge transfer for neutral/ion collision pairs. The total number of candidate collision partners within a cell is determined using Bird's No-Time-Counter (NTC) ${ }^{9}$ method. The probability of a collision event is then determined for these candidate pairs based on the total collision cross section. MEX collisions for neutral particle interactions are modeled using variable hard sphere $^{9}$ (VHS) total cross sections and isotropic scattering. 
The corresponding VHS parameters including the reference diameter $d_{r e f}$, reference temperature $T_{r e f}$, and temperature exponent $\omega$ for the gas mixture constituents are provided in Table 1.

Table 1. VHS parameters for $\mathrm{H}_{2} / \mathrm{N}_{2} / \mathrm{NH}_{3}$

\begin{tabular}{|c|c|c|c|}
\hline & $d_{\text {ref }}$ & $T_{\text {ref }}$ & $\omega$ \\
\hline $\mathrm{H}_{2}$ & $2.92 \AA$ & $273 \mathrm{~K}$ & 0.75 \\
$\mathrm{~N}_{2}$ & $4.17 \AA$ & $273 \mathrm{~K}$ & 0.75 \\
$\mathrm{NH}_{3}$ & $5.94 \AA$ & $273 \mathrm{~K}$ & 0.75 \\
\hline
\end{tabular}

Post-collision velocities involving neutral collision pairs are assumed to follow isotropic scattering in the center-of-mass frame of reference. Collisions involving neutral/ion pairs, however, scatter anisotropically, with a strong forward-scattering tendency. Total and differential cross section data for this system are obtained from the literature ${ }^{12-14}$ for both direct and charge transfer collisions based on guided ion beam experimental results as well as the electron nuclear dynamics formalism. While the total cross section is invariant under transformation of reference frame, the differential cross section must be converted from the laboratory $(\mathrm{LAB})$ reference frame to the center-of-mass $(\mathrm{CM})$ reference frame for use within the DSMC/PIC framework. The differential cross section (DCS) transformation is established through the relationship between the LAB and CM total cross-sectional area:

$$
\frac{d \sigma\left(\theta_{C M}\right)}{d \Omega_{C M}} d \Omega_{C M}=\frac{d \sigma\left(\theta_{L A B}\right)}{d \Omega_{L A B}} d \Omega_{L A B}
$$

where:

$$
\begin{gathered}
d \Omega_{C M}=2 \pi \sin \left(\theta_{C M}\right) d \theta_{C M}, \\
d \Omega_{L A B}=2 \pi \sin \left(\theta_{L A B}\right) d \theta_{L A B} .
\end{gathered}
$$

For a $\mathrm{LAB} \rightarrow \mathrm{CM}$ transformation, Eq 2 is written as:

$$
\frac{d \sigma\left(\theta_{C M}\right)}{d \Omega_{C M}}=\frac{d \sigma\left(\theta_{L A B}\right)}{d \Omega_{L A B}} \frac{d \Omega_{L A B}}{d \Omega_{C M}}=\frac{d \sigma\left(\theta_{L A B}\right)}{d \Omega_{L A B}} \frac{\sin \left(\theta_{L A B}\right) d \theta_{L A B}}{\sin \left(\theta_{C M}\right) d \theta_{C M}}
$$

The transformation is established by obtaining an analytical expression (if possible) for the term on the far right hand side of Eq 5. The relationship between $\theta_{L A B}$ and $\theta_{C M}$ may be determined by considering the schematic of scattering of two particles $A$ and $B$ in the center-of-mass (CM) frame of reference shown in Figure 1. If the particle $\mathrm{B}$ is considered to be at rest in the laboratory frame of reference, then the point $\mathrm{B}$ lies on the dashed circle with a radius $p_{0}=\mu v$, where $\mu=m_{1} m_{2} /\left(m_{1}+m_{2}\right)$ is the reduced mass and $v=v_{1}-v_{2}$ is the relative velocity prior to collision. In the case when $m_{1}=m_{2}$, both $\mathrm{A}$ and $\mathrm{B}$ lie on the dashed circle, and it is straight-forward to show that the relationship between $\theta_{L A B}$ and $\theta_{C M}$ is:

$$
\theta_{C M}=2 \theta_{L A B}
$$

resulting in the differential cross section transformation for direct or (resonant) charge transfer collisions:

$$
\frac{d \sigma\left(\theta_{L A B}\right)}{d \Omega_{L A B}}=4 \cos \theta_{L A B} \frac{d \sigma\left(\theta_{C M}\right)}{d \Omega_{C M}}
$$

In the gas mixture, it is necessary to account for cases where $\mathrm{m}_{1} \neq \mathrm{m}_{2}$. To simplify the analysis, we assume that $\mathrm{v}_{1} \gg \mathrm{v}_{2}$ such that the second particle is at rest, and point $\mathrm{B}$ thus lies on the dashed circle as in Figure 1. This is a reasonable assumption at GEO, since the $\mathrm{H}^{+}$are approximately $1 \mathrm{keV}$, and the hydrazine chemical species are characterized by energies of less than $1 \mathrm{eV}$. Following this approach then, we have that $\mathrm{m}_{1}$ corresponds to $\mathrm{m}_{H^{+}}$, and $m_{1}<m_{2}$ regardless of the collision partner for all chemical species in the 


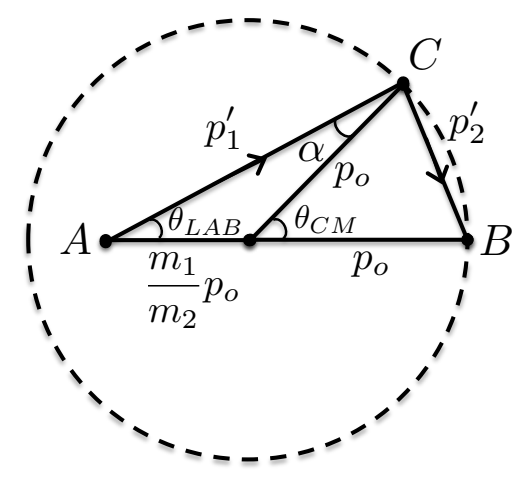

Figure 1. Transformation from $\mathrm{LAB} \rightarrow \mathrm{CM}$ frame of reference for particles of different mass.

hydrazine mixture, as shown in Figure 1.

Invoking the sum of angles and law of sines, we can establish the relationship between the scattering angle of particle $1\left(\mathrm{H}^{+}\right.$in this case) in the LAB frame $\left(\theta_{L A B}\right)$ and the $\mathrm{CM}$ frame $\left(\theta_{C M}\right)$, and finally the expressions for $\sin \theta_{L A B}$ and $d \theta_{L A B}$ which are required for the transformation:

$$
\begin{aligned}
\theta_{C M} & =\theta_{L A B}+\sin ^{-1}\left(\frac{m_{1}}{m_{2}} \sin \theta_{L A B}\right) \\
d \theta_{C M} & =1+\frac{m_{1} / m_{2} \cos \theta_{L A B}}{\sqrt{1-\left(m_{1} / m_{2}\right)^{2} \sin ^{2} \theta_{L A B}}} \\
\sin \theta_{C M} & =\sin \left[\theta_{L A B}+\sin ^{-1}\left(\frac{m_{1}}{m_{2}} \sin \theta_{L A B}\right)\right]
\end{aligned}
$$

The transformation can be made by expressing the right hand side of Equation 5 in terms of $\theta_{C M}$ and then plotting the CM DCS as a function of $\theta_{C M}$. However, Equations 8 - 10 can only be solved approximately using an iterative solution approach, so an immediate analytical form is not possible. If instead the right hand side is left in terms of $\theta_{L A B}$, the final $\mathrm{LAB} \rightarrow \mathrm{CM}$ transformation is expressed as:

$$
\frac{d \sigma\left(\theta_{C M}\right)}{d \Omega_{C M}}=\frac{\sin \theta_{L A B}}{\sin \left[\theta_{L A B}+\sin ^{-1}\left(\frac{m_{1}}{m_{2}} \sin \theta_{L A B}\right)\right]\left(1+\frac{m_{1} / m_{2} \cos \theta_{L A B}}{\sqrt{1-\left(m_{1} / m_{2}\right)^{2} \sin ^{2} \theta_{L A B}}}\right)} \frac{d \sigma\left(\theta_{L A B}\right)}{d \Omega_{L A B}}
$$

The right hand side is evaluated with a value for the LAB DCS and the corresponding angle $\theta_{L A B}$ (from experimental data), and finally the transformed value for the CM DCS is obtained. These values are plotted against $\theta_{C M}$, which is determined for each value of $\theta_{L A B}$ from Equation 8. This transformation is applied to the differential cross sections describing the post-collision scattering of $\mathrm{H}_{2}-\mathrm{H}^{+}, \mathrm{N}_{2}-\mathrm{H}^{+}$and $\mathrm{NH}_{3}-\mathrm{H}^{+}$ collision partners in the center-of-mass frame of reference, and the resulting cross sections are plotted in Figure 2(a). The $\mathrm{LAB} \rightarrow \mathrm{CM}$ transformation is most prominent for cases in which the collision partners are of equal mass $\left(m_{1}=m_{2}\right)$, and the differential cross section in the CM frame of reference approaches the LAB frame value when $m_{1}<<m_{2}$. Total direct (non-transfer) and charge transfer cross sections employed in this model are reported in the literature for the mixture constituents, ${ }^{12-14}$ and the charge transfer cross sections are shown in Figure 2(b).

\section{Magnetic Field Model}

In addition to CEX interactions, charged particles in GEO are subject to interaction with Earth's magnetic field. The interaction of spacecraft ions with the magnetic field plays an important role in the evolution of the ion plume. Both the strength of the magnetic field and the orientation of the field lines relative to the plume 


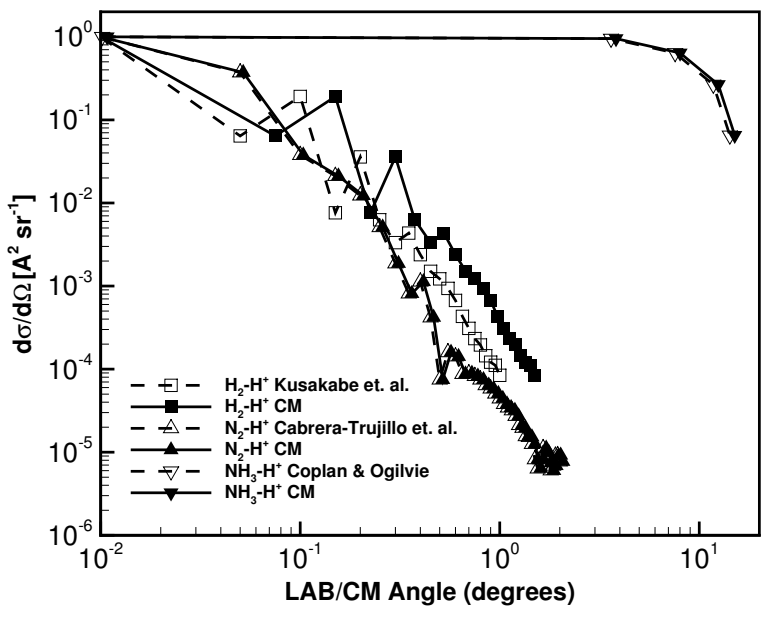

(a) DCS used for $\mathrm{H}_{2} / \mathrm{N}_{2} / \mathrm{NH}_{3}-\mathrm{H}^{+}$system.



(b) TCS used for $\mathrm{H}_{2} / \mathrm{N}_{2} / \mathrm{NH}_{3}-\mathrm{H}^{+}$system.

Figure 2. (a) Differential cross sections (DCS) and (b) total cross sections (TCS) for collision dynamics of $\mathrm{H}_{2} / \mathrm{N}_{2} / \mathrm{NH}_{3}-\mathrm{H}^{+}$system.

propagation have a substantial impact on the development of the plume, as magnetization of spacecraft ions can impede ion flow in the axial and radial directions. The magnetic field model developed for this work investigates the impact of magnetic field strength on the spacecraft ion plume formation assuming a fixed field line orientation relative to the spacecraft.

Immediately after a CEX event, the newly formed ion enters into a gyroscopic orbit about a magnetic field line. This orbit is characterized by the Larmor radius, $r_{L}$, and the gyration frequency, $\omega_{L}$, which are determined according to:

$$
\begin{gathered}
r_{L}=\frac{V_{x 0}^{i o n}}{\omega_{L}}, \\
\omega_{L}=\frac{q^{i o n} B}{m^{i o n}} .
\end{gathered}
$$

In Eq. $12, V_{x 0}^{\text {ion }}$ is the initial x-velocity of the $\mathrm{H}_{2}^{+} / \mathrm{N}_{2}^{+} / \mathrm{NH}_{3}^{+}$species entering the gyro-orbit, which is equivalent to the post-collision x-velocity after a charge exchange reaction. In Eq. 13, $q^{\text {ion }}$ is the fundamental charge, $B$ is the magnetic field strength, and $m^{i o n}$ is the molecular mass of the $\mathrm{H}_{2}^{+} / \mathrm{N}_{2}^{+} / \mathrm{NH}_{3}^{+}$species. From these expressions, it is clear that the magnetic field strength uniquely determines the gyration frequency for a given charged chemical species. The Larmor radius, however, is dependent on both magnetic field strength (through $\omega_{L}$ ) as well as on the translational energy of the magnetized ion orthogonal to the field line.

Within the present axisymmetric simulations, the magnetic field lines are assumed to be oriented vertically (parallel to the $z$-axis, shown by dashed lines in Figure 3 ). Thus, only the $x$-velocity component of the magnetized ions follows a gyroscopic motion, while the $z$-velocity component is unimpeded by the magnetic field. Magnetized ions entering a gyroscopic orbit with a non-zero velocity component along the field line thus follow a helical trajectory. The frame of reference is held fixed to the spacecraft thruster at the origin, such that the ambient flow, and hence the geomagnetic field lines, have a velocity equivalent to the orbital velocity relative to the spacecraft. The gyroscopic motion due to the magnetic field is imposed on the ions through a time-dependent velocity, which for a constant magnetic field aligned with the $z$-axis, is determined according to:

$$
V_{x}^{i o n}=V_{B} \pm\left(V_{x 0}^{i o n}-V_{B}\right) \sin \left(\frac{q^{i o n} B}{m^{i o n}} t+\phi^{i o n}\right)
$$




$$
\phi^{i o n}= \begin{cases}\pi / 2 & \text { if } V_{x o}^{i o n}<V_{B} \\ 3 \pi / 2 & \text { if } V_{x 0}^{i o n}>V_{B}\end{cases}
$$

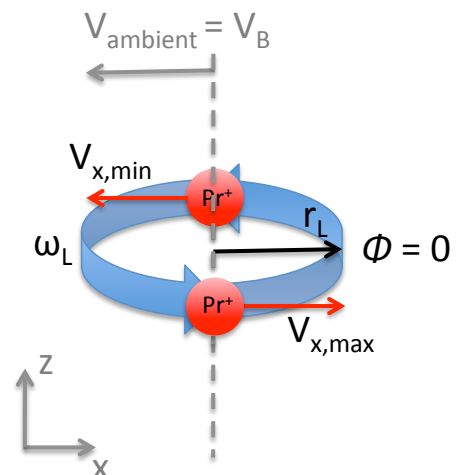

(a) Magnetized spacecraft propellant ion.

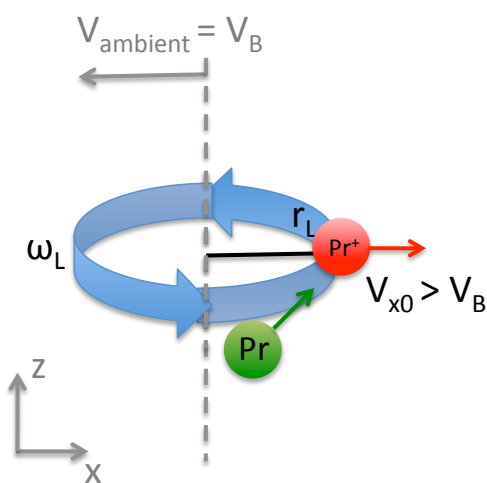

(b) Spacecraft propellant neutral/ion collision resulting in MEX and new guiding center for propellant ion.

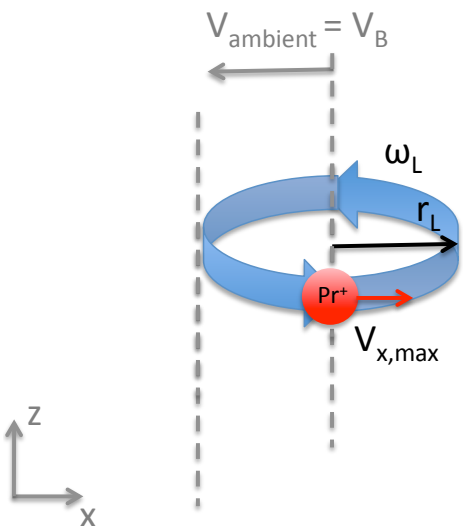

(c) Magnetized spacecraft propellant ion on new guiding center.

Figure 3. Schematic of cross-field diffusion mechanism incorporated in magnetic field model for $B=1.1 \times 10^{-7}$ T. (a) Magnetized spacecraft propellant ion (where $\mathrm{Pr}^{+}$represents any of $\mathbf{H}_{2}^{+}, \mathrm{N}_{2}^{+}, \mathrm{NH}_{3}^{+}$) with gyroscopic motion about magnetic field line undergoes MEX collision (b) with spacecraft propellant neutral. (c) Postcollision velocity of spacecraft propellant ion is used to update the phase angle, which defines new guiding center.

The gyroscopic term on the right-hand side is added when $\phi_{\text {ion }}=3 \pi / 2$ and subtracted when $\phi_{i o n}=\pi / 2$. The velocity $V_{x 0}^{i o n}$ is the initial velocity of the magnetized ion as it enters the gyro-orbit, which is assumed equal to the post-collision velocity of a spacecraft propellant ion formed through CEX. When the ion enters the orbit, the phase angle, $\phi^{i o n}$, is specified according to the relative velocity of the ion with respect to the magnetic field line velocity, $V_{B}$. This is shown schematically in Figure 3(a), for a case in which the ambient flow (and therefore the field line velocity) is directed to the left. If the ion has a velocity to the left relative to the field line, the ion enters the orbit with a phase of $\phi^{i o n}=\pi / 2$. This represents a minimum orbital velocity, and the ion velocity is thus $V_{x 0}=V_{x, \min }$. If the ion has a velocity to the right relative to the field line, the ion enters the orbit with a phase of $\phi^{i o n}=3 \pi / 2$, and the ion velocity corresponds to $V_{x 0}=V_{x, \max }$. The time $t$ in Eq.(14) is initialized to zero when the ion enters the orbit, and is advanced by the simulation timestep. The gyration frequency in Eq.(13) is species-dependent, and has a values of $\omega_{L, H_{2}}=0.87, \omega_{L, N_{2}}=0.06$ and $\omega_{L, N H_{3}}=0.1$ revolutions per second, for $H_{2}, N_{2}$ and $N_{3}$, respectively. The timestep used in the simulations is $d t=2.5 \times 10^{-4} \mathrm{~s}$, and thus a single orbit is resolved by $\mathbf{O}\left(10^{3}\right)$ simulation timesteps.

Once magnetized, the motion of the spacecraft propellant ions is a superposition of the magnetic field velocity and the unsteady gyration velocity in the $x$-direction. In a collisional flow, however, these magnetized ions may undergo collisions with other particles. A collision resulting in both momentum and charge exchange would effectively 'demagnetize' the spacecraft propellant ion, and the resulting propellant neutral would follow a linear trajectory according to its post-collision velocity. This demagnetization process is modeled for the spacecraft constituent charge transfer only.

It is also possible for a collision between a magnetized propellant ion and a neutral particle to result in momentum exchange only. Although the spacecraft ion keeps the charge and remains magnetized, the momentum exchange will effectively bump the ions onto a new orbit with a new guiding center. This process is shown schematically in Figures 3(b) and 3(c), in which a magnetized spacecraft ion undergoes a MEX collision with an neutral spacecraft particle. In this particular scenario, the spacecraft ion velocity has a phase $\phi^{i o n}=0$, and thus has zero velocity relative to the field line guiding center. Upon collision, the momentum transfer will result in a finite post-collision $x$-velocity component (e.g., to the right as shown by the red arrow). This post-collision velocity defines the initial velocity $V_{x 0}^{i o n}$ for a different orbit about a new 
guiding center, shown in Figure 3(c). To define the guiding center of the new orbit, the phase angle $\phi^{i o n}$ must be evaluated after each MEX collision using the velocity criteria specified in Eq. (15), where $V_{x 0}^{i o n}$ is taken as the spacecraft ion post-collision velocity.

This treatment of MEX collisions involving magnetized spacecraft ions provides a mechanism for crossfield diffusion of the spacecraft ions within these simulations. As mentioned in Section $\mathrm{B}$, the ambient $\mathrm{H}^{+}$ ions are modeled as being trapped indefinitely on the magnetic field lines to preserve the ambient conditions. In consideration of the gyroscopic parameters in Eqs.(12) and (13), the $\mathrm{H}^{+}$ions are modeled as magnetized on field lines of infinite strength, $B \rightarrow \infty$. The influence of the magnetic field on the spacecraft ions, $\mathrm{H}_{2}^{+}, \mathrm{N}_{2}^{+}$and $\mathrm{NH}_{3}^{+}$, is examined in this work by considering the case of $B=1.1 \times 10^{-7} \mathrm{~T}$ found in GEO, and the limiting case of $B \rightarrow 0$.

\section{Plume Configurations}

The plume flow examined in this study involves the steady firing of a spacecraft thruster into the ambient magnetosphere free stream in GEO. The flow is simulated on an axi-symmetric spherical computational domain shown in Figure 4, with a radius of $35 \mathrm{~km}$ and an axis of symmetry along the $x$-axis. The thruster considered in this study is located at the origin $(x, z)=0$, and generates thrust in the $-x-$ direction, such that the plume flow is initially directed in the $+x$-direction (indicated by the red arrow in Figure 4 ). An inflow boundary condition is specified at the nozzle exit plane (not visible in Figure 4), with a nominal mass flow rate of $\dot{m}=5.0 \times 10^{-4} \mathrm{~kg} / \mathrm{s}$. The plume at the exit plane is modeled as a charge-neutral propellant mixture of molecular hydrogen, nitrogen and ammonia, with the properties shown in Table 2. The simulation domain is held fixed with respect to the spacecraft thruster, so the ambient $\mathrm{H}^{+}$ions follow a thermal distribution in this frame of reference. Recalling discussion of the magnetic field model, the geostationary magnetic field lines are assumed to be aligned vertically in these figures, and are stationary relative to the spacecraft thruster.

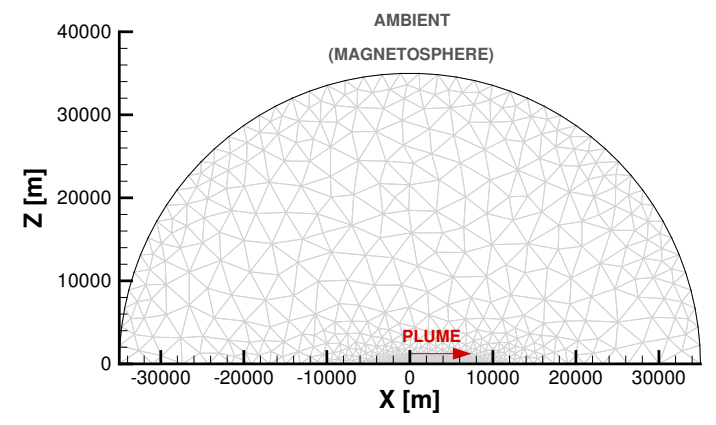

Figure 4. DSMC/PIC computational domain. Axis of symmetry lies along the x-axis, and the nozzle exit plane is located at the origin. Red arrow indicates the plume flow direction, and geomagnetic field lines are aligned with the z-axis.

Table 2. Plume and Ambient Flow Conditions

\begin{tabular}{|c||c|c|c|c|}
\hline Species & $\mathrm{m}\left[\mathrm{kg} \mathrm{kmol}{ }^{-1}\right]$ & $\mathrm{k}_{\mathrm{b}} \mathrm{T} / \mathrm{q}_{\mathrm{o}}[\mathrm{eV}]$ & $\mathrm{V}\left[\mathrm{m} \mathrm{s}^{-1}\right]$ & $\mathrm{n}\left[\mathrm{m}^{-3}\right]$ \\
\hline $\mathrm{H}_{2}$ & 2.02 & 0.03 & 1900 & $3.99 \times 10^{23}$ \\
$\mathrm{~N}_{2}$ & 28.0 & 0.03 & 1900 & $1.74 \times 10^{22}$ \\
$\mathrm{NH}_{3}$ & 17.0 & 0.03 & 1900 & $1.99 \times 10^{22}$ \\
\hline $\mathrm{H}^{+}$ & 1.01 & $1.0 \times 10^{3}$ & 0 & $3.0 \times 10^{6}$ \\
\hline
\end{tabular}

Typical values of the spacecraft propellant ion gyroscopic radii are summarized in Table 3 , based on a nominal magnetic field strength of $\mathrm{B}=1.1 \times 10^{-7} \mathrm{~T}$ including diurnal variation. ${ }^{15}$ The ambient/inflow 
Table 3. Larmor Radius (with B-field variation)

\begin{tabular}{|c||c|c|}
\hline Species & mean $[\mathrm{km}]$ & variation $(+/-)$ \\
\hline $\mathrm{r}_{L, H_{2}}$ & 0.4 & $0.30 / 0.46$ \\
$\mathrm{r}_{L, N_{2}}$ & 5.6 & $4.2 / 6.5$ \\
$\mathrm{r}_{L, N H_{3}}$ & 3.3 & $2.6 / 4.0$ \\
\hline
\end{tabular}

properties of temperature, velocity and number density are specified only for the CEX reactant species initialized as ambient $\left(H^{+}\right)$or at the inflow (neutral species) boundary.

\section{Results}

In this section, steady-state plume results are presented first. In particular, the influence of the magnetic field on the development of both the spacecraft neutral and ion plumes is investigated. The first case that is presented examines the ion and neutral plume development in the absence of the geostationary magnetic field. This allows for an assessment of the electrostatic field forces in the GEO environment. The second case incorporates the influence of the magnetic field in addition to the self-consistent electric field. Recall that only the $\mathrm{H}_{2}^{+} / \mathrm{N}_{2}^{+} / \mathrm{NH}_{3}^{+}$ions become magnetized, thus the magnetic field does not directly impact the motion of the neutral $\mathrm{H}_{2} / \mathrm{N}_{2} / \mathrm{NH}_{3}$ particles. It is important to note, however, that the magnetized ions may become demagnetized through CEX reactions, and so the influence of the magnetic field on the development of the neutral plumes is examined as well.

The second set of simulation results examine an unsteady thruster firing, in which the thrusters are fired for 2 seconds and then turned off. The evolution of the neutral plume is examined during and after the thruster burn to determine the rate of dissipation of each chemical species from the computational domain.

\section{A. Plume Development without Magnetic Field $(B=0)$}

The steady-state flow field in the absence of a magnetic field is presented in Figures 5-7. The contours in Figures 5-7(a) represent the number density of the spacecraft neutrals which are emitted from the thruster with a bulk velocity in the $+x$-direction. As the neutral plume expands outward from the thruster, the particles may undergo MEX and CEX collisions with the ambient particles as well as with each other. The number density of the corresponding spacecraft ions generated through charge exchange are shown in 5-7(b). Both neutral and ion plumes undergo expansion in the near-vacuum ambient, and the ion plume is also subject to forces from the self-consistent electric field.

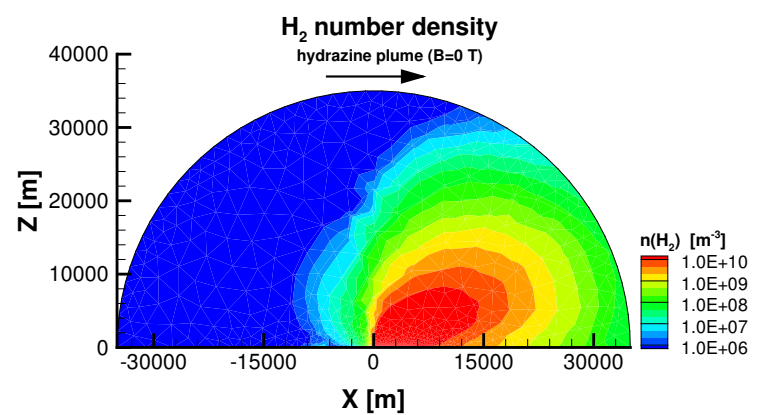

(a) Contours of $\mathrm{H}_{2}$ number density, $\mathrm{B}=0 \mathrm{~T}$.

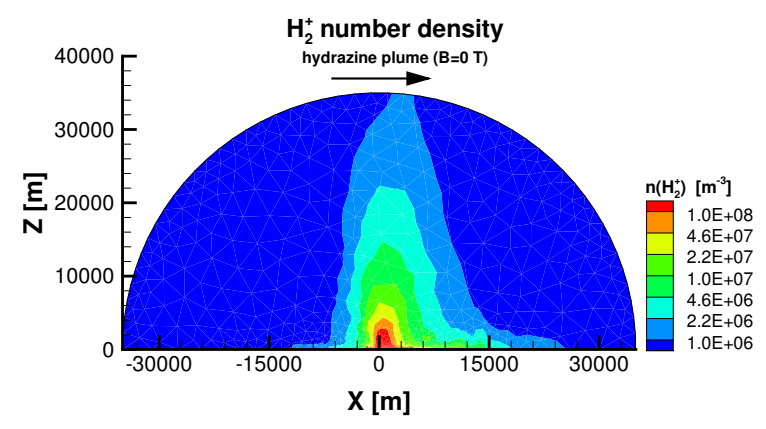

(b) Contours of $\mathrm{H}_{2}^{+}$number density, $\mathrm{B}=0 \mathrm{~T}$.

Figure 5. Contours of (a) $\mathbf{H}_{2}$ number density and (b) $\mathbf{H}_{2}^{+}$number density for $+x-$ flow configuration. The lower saturation limit on the contour levels correspond to the ambient density. Note the difference in the contour level upper limits.

Comparison of the neutral and ion plumes in Figures 5-7 reveals the influence of the self-consistent electrostatic field on the ion plume. The neutral chemical species plume undergoes considerable expansion, 


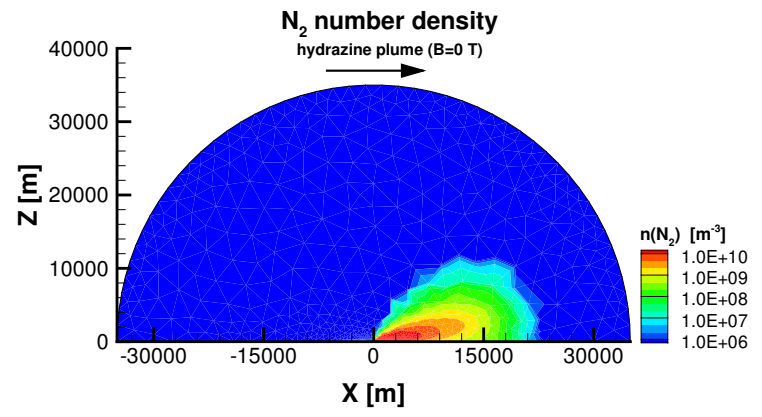

(a) Contours of $\mathrm{N}_{2}$ number density, $\mathrm{B}=0 \mathrm{~T}$.

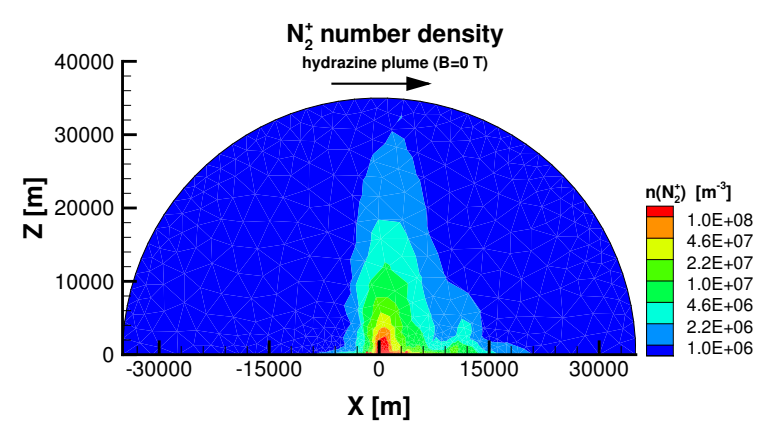

(b) Contours of $\mathrm{N}_{2}^{+}$number density, $\mathrm{B}=0 \mathrm{~T}$.

Figure 6. Contours of (a) $\mathbf{N}_{2}$ number density and (b) $\mathbf{N}_{2}^{+}$number density for $+x-$ flow configuration. The lower saturation limit on the contour levels correspond to the ambient density. Note the difference in the contour level upper limits.

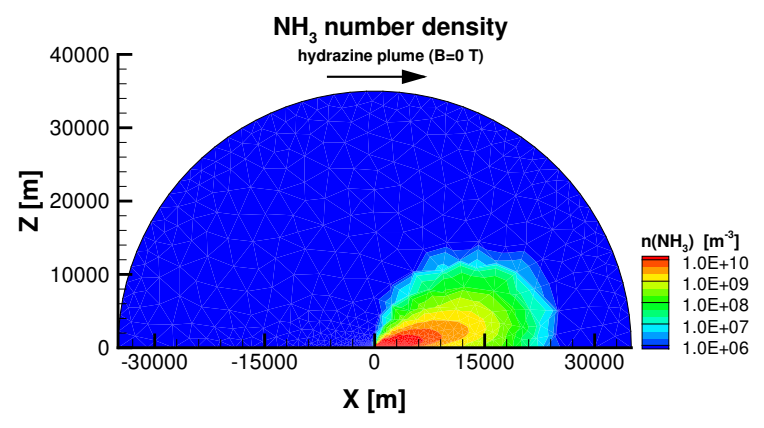

(a) Contours of $\mathrm{NH}_{3}$ number density, $\mathrm{B}=0 \mathrm{~T}$.

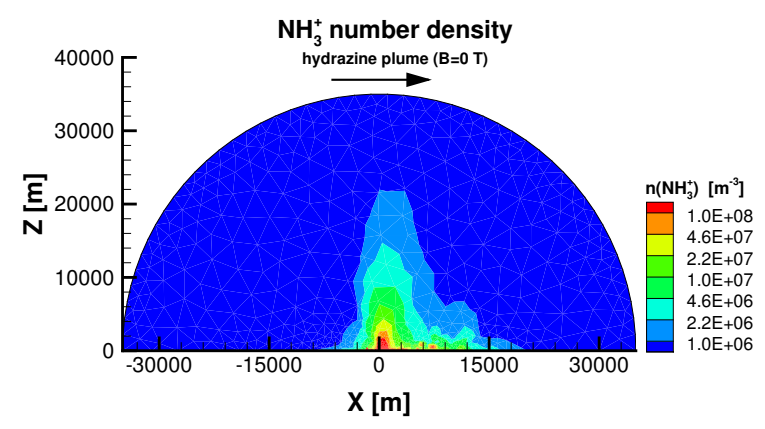

(b) Contours of $\mathrm{NH}_{3}^{+}$number density, $\mathrm{B}=0 \mathrm{~T}$.

Figure 7. Contours of (a) $\mathrm{NH}_{3}$ number density and (b) $\mathrm{NH}_{3}^{+}$number density for $+x$-flow configuration. The lower saturation limit on the contour levels correspond to the ambient density. Note the difference in the contour level upper limits.

but the plume generally propagates in the $+x$-direction. The plume concentrations in the farfield remain orders of magnitude higher than the ambient density, which is represented by the lower saturation of the contour levels at $1.0 \times 10^{6} \mathrm{~m}^{-3}$. Owing to the plume composition and relatively small molecular mass, the $\mathrm{H}_{2}$ plume shows the greatest extent while the $\mathrm{N}_{2}$ and $\mathrm{NH}_{3}$ plumes are at lower concentrations.

The corresponding ion plumes are siginficantly lower in density, although the concentration is still an order of magnitude higher than the ambient density at distances of $10 \mathrm{~km}$ from the thruster origin. The evolution of the ion plumes indicates a strong influence of the electric field on the charged chemical speices. The acceleration due to the electrostatic forces drive the ions away from the thruster origin, both in the upstream $(-x)$ and downstream $(+x)$ directions. In each case, the ion plume concentration is slightly higher in the downstream direction, resulting in an asymmetry of the ion plumes about $x=0$; this is attributed to the higher concentration of neutral plume chemical species downstream which may undergo charge exchange. This asymmetry, however, is quite subtle, which underscores the influence of the electrostatic field on the ion plumes.

\section{B. Plume Development with Magnetic Field $\left(B=1.1 \times 10^{-7} \mathbf{T}\right)$}

The steady-state flow field generated in the presence of the geostationary magnetic field is presented in Figures 8-10(a) and Figures 8-10(b). The contours in Figures 8-10(a) again represent the number density of the spacecraft neutrals which are emitted from the thruster. Recall that these spacecraft neutrals may undergo charge exchange with the ambient $\mathrm{H}^{+}$ions, and then subsequently enter into gyroscopic orbit about 
the geomagnetic field lines which are stationary in this frame of reference. The number density of the spacecraft ions generated through charge exchange are shown in Figures 8-10(b). The development of both the neutral plume and ion plume in the presence of the magnetic field shows some general similarities to the results in the previous section. The neutral plumes again develop largely in the $+x$-direction, as the electromagnetic field does not directly influence the neutral particles. The ion plume, however, becomes confined by the stationary magnetic field lines and exhibits a peaked cone-like structure centered near $x=0$.

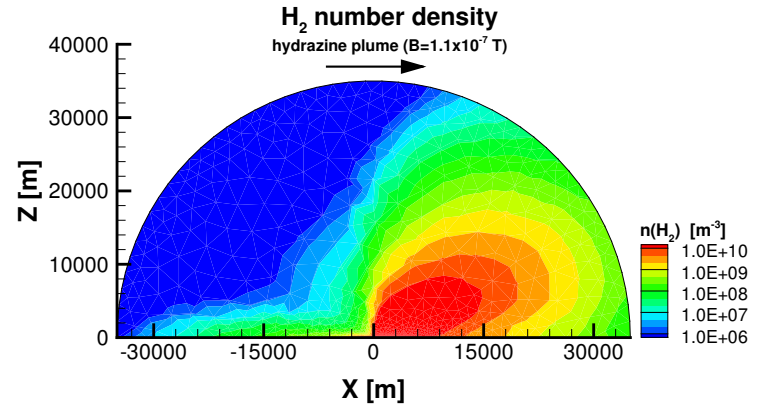

(a) Contours of $\mathrm{H}_{2}$ number density, $\mathrm{B}=1.1 \times 10^{-7} \mathrm{~T}$.

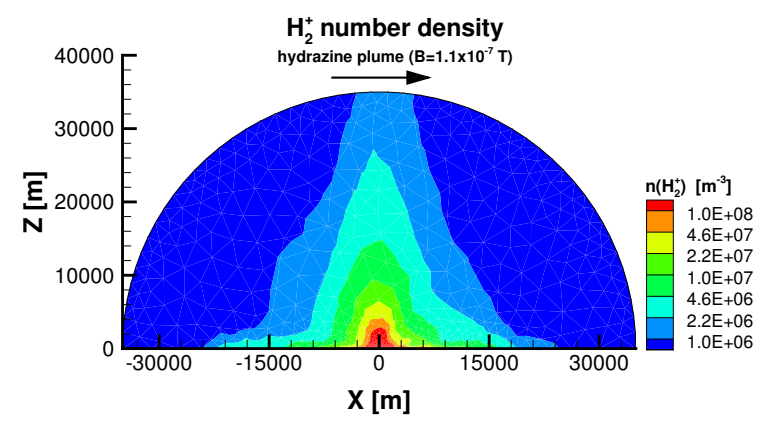

(b) Contours of $\mathrm{H}_{2}^{+}$number density, $\mathrm{B}=1.1 \times 10^{-7} \mathrm{~T}$.

Figure 8. Contours of (a) $\mathbf{H}_{2}$ number density and (b) $\mathbf{H}_{2}^{+}$number density for $+x-$ flow configuration. The magnetic field model accounts for the gyroscopic motion of magnetized ions. The lower saturation limit on the contour levels correspond to the ambient density. Note the difference in the contour level upper limits.

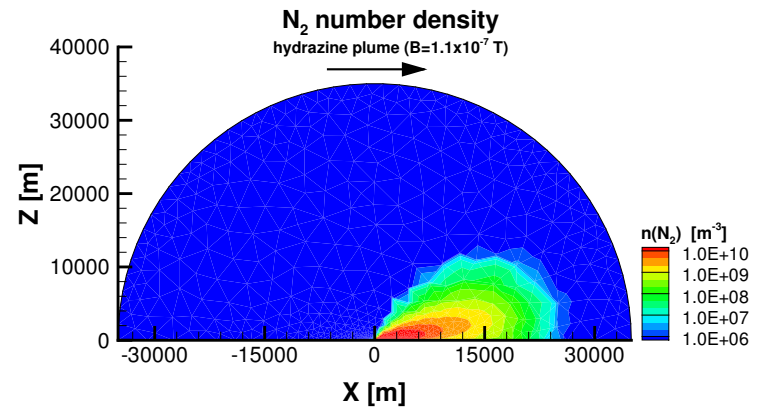

(a) Contours of $\mathrm{N}_{2}$ number density, $\mathrm{B}=1.1 \times 10^{-7} \mathrm{~T}$.

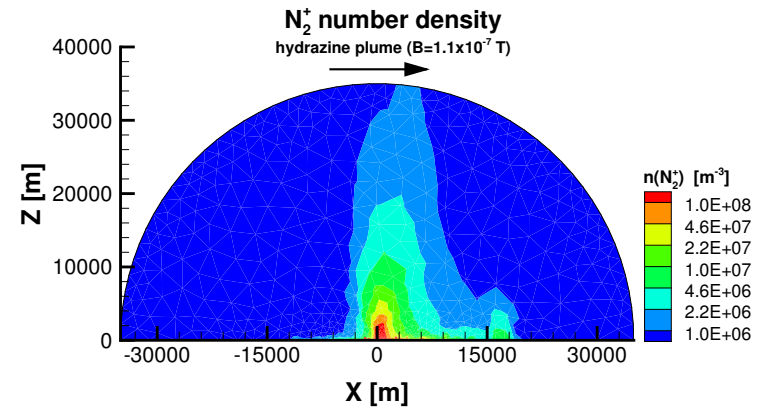

(b) Contours of $\mathrm{N}_{2}^{+}$number density, $\mathrm{B}=1.1 \times 10^{-7} \mathrm{~T}$.

Figure 9. Contours of (a) $\mathbf{N}_{2}$ number density and (b) $\mathbf{N}_{2}^{+}$number density for $+x-$ flow configuration. The magnetic field model accounts for the gyroscopic motion of magnetized ions. The lower saturation limit on the contour levels correspond to the ambient density. Note the difference in the contour level upper limits.

When the ions are formed through charge exchange, the particles immediately enter into a gyroscopic orbit about the stationary magnetic field lines, which are oriented parallel to the $z$-axis. This effectively modifies the development of the ion plume in the $\pm x$-direction by confining the ion motion to within the Larmor radius. At the same time, however, the ions are subject to the electrostatic field forces, which act in directions orthogonal to and parallel to the magnetic field lines. The component of the electrostatic force orthogonal to the field lines effectively pushes the ion onto a new guiding center, resulting in a diffusion of the ion species across the field lines. This diffusion or spreading of the ion plume is most prominent near the thruster (at small $z$-distance). At larger distances, the plume-induced field strength attenuates and the spreading of the plume diminishes. Ion motion along the magnetic field lines is unimpeded, and the components of the electrostatic forces parallel to the field lines accelerate ions vertically away from the thruster, resulting in a cone-like plume structure. Although the ions enter into gyroscopic motion about stationary magnetic field lines (or guiding centers), the acceleration of these ions due to the self-consistent electric field competes with the gyroscopic acceleration. Thus the ion plumes show a distinct stucture in the presence of the magnetic field. The asymmetry of the ion plumes is also observed in these contours, owing 


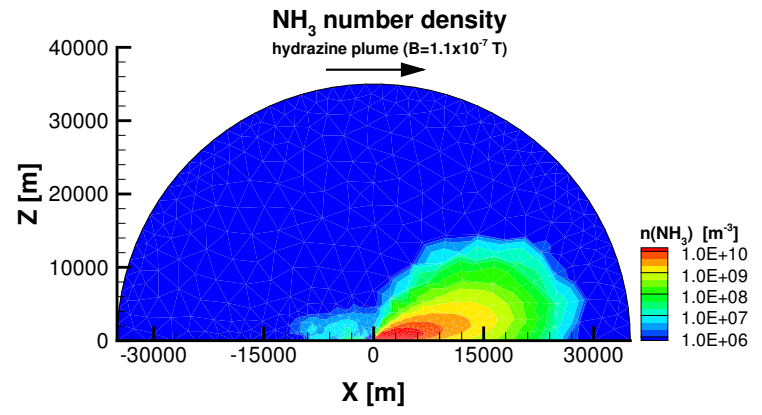

(a) Contours of $\mathrm{NH}_{3}$ number density, $\mathrm{B}=1.1 \times 10^{-7} \mathrm{~T}$.

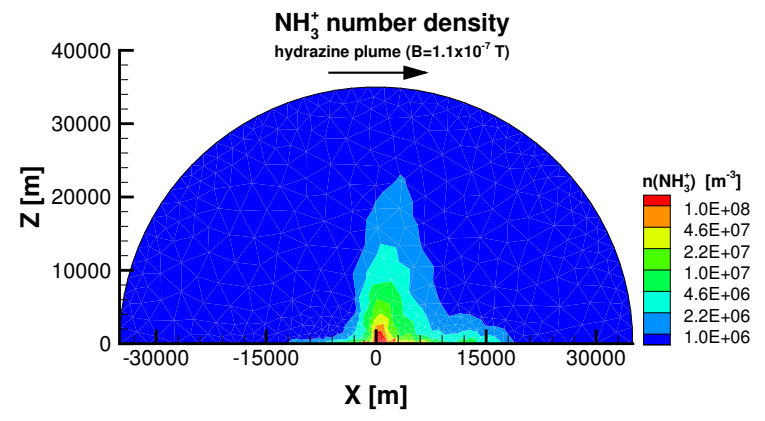

(b) Contours of $\mathrm{NH}_{3}^{+}$number density, $\mathrm{B}=1.1 \times 10^{-7} \mathrm{~T}$.

Figure 10. Contours of (a) $\mathrm{NH}_{3}$ number density and (b) $\mathrm{NH}_{3}^{+}$number density for $+x-$ flow configuration. The magnetic field model accounts for the gyroscopic motion of magnetized ions. The lower saturation limit on the contour levels correspond to the ambient density. Note the difference in the contour level upper limits.

to the enhanced neutral plume concentration in the $+x$-direction.

\section{Unsteady plume simulations}

Plume simulation results are presented next which examine an unsteady thruster plume firing for two seconds, after which the thrusters are turned off. The thrusters fire in the $+x$-direction, and the neutral plume expands in the near-vacuum ambient while undergoing charge exchange with the ambient ions. The simulation results show the convection and eventual dissipation of the neutral plume to levels below the ambient density of approximately $1 \times 10^{6} \mathrm{~m}^{-3}$ for each of the chemical species. Each column in Figure 11 represents the time evolution of the $\mathrm{H}_{2}, \mathrm{~N}_{2}$ and $\mathrm{NH}_{3}$ neutral plumes, starting at two seconds (top row) and stepping through time up until the majority of the plume has convected out of the domain (bottom row).

The total time required for the neutral plume to dissipate to the ambient density everywhere $( \pm 35 \mathrm{~km}$ from the spacecraft) is approximately 34 seconds. The core of the neutral plume (the highest density region) leaves the computational domain after approximately 15 seconds, which is the time required for a plume traveling at the thruster exit velocity of $1900 \mathrm{~m} / \mathrm{s}$ to reach the edge of the computational domain. As the plume is comprised of chemical species which vary in molecular weight, the lighter plume species are faster and the core of the $\mathrm{H}_{2}$ plume reaches the edge of the domain first, followed by the $\mathrm{NH}_{3}$ and then the $\mathrm{N}_{2}$ plume core. This 'time of flight' is nearly half of the total dissipation time. The additional time required for dissipation is largely a result of the plume expansion, as well as collisional effects near the thruster exit. These mechanisms work to decrease or reverse the plume neutral particle velocities such that the edges of the plume remain within the computational domain for an extended period after the core of the plume has already vanished. It is found that the $\mathrm{N}_{2}$ plume vanishes completely (below the ambient number density levels) first, after approximately 26 seconds, while the $\mathrm{H}_{2}$ is the final chemical species to dissipate completely.

\section{Conclusions}

The focus of this work was to examine the development of spacecraft neutral and ion plumes during steady and unsteady hydrazine thruster burns. The plume was modeled as a gas mixture comprised of the primary hydrazine post-combustion products, consisting of $\mathrm{H}_{2}, \mathrm{~N}_{2}$ and $\mathrm{NH}_{3}$, and these chemical species interacted with the ambient $\mathrm{H}^{+}$ions in the magnetosphere. Detailed differential and total cross sections were used to model the formation of spacecraft ions formed through charge exchange (CEX) reactions with the ambient magnetosphere plasma. Also of interest was the influence of the geomagnetic field on the ion plume. A combined DSMC/PIC methodology was used to properly capture the non-equilibrium collisional and plasma phenomena that are relevant in the rarefied plasma environment in GEO. The development of the steady spacecraft neutral and ion plume was examined under two different magnetic field configurations, assuming a magnetic field strength of $\mathrm{B}=0 \mathrm{~T}$ and $\mathrm{B}=1.1 \times 10^{-7} \mathrm{~T}$.

The development of the neutral mixture plume was not strongly influenced by the presence of the elec- 


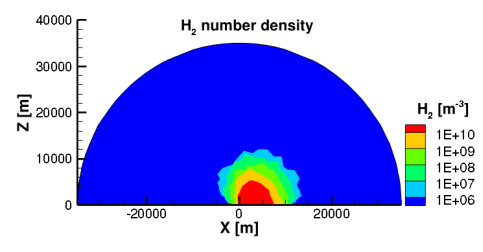

(a) $\mathrm{H}_{2}$ number density, $2.0 \mathrm{~s}$

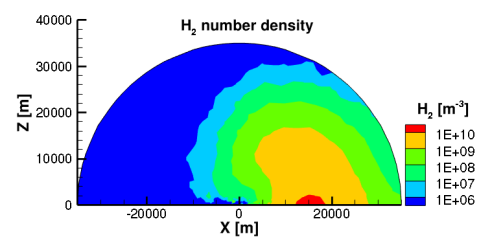

(d) $\mathrm{H}_{2}$ number density, $6.5 \mathrm{~s}$

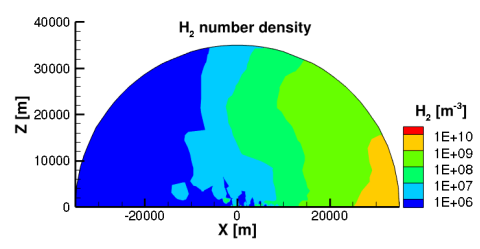

(g) $\mathrm{H}_{2}$ number density, $12.5 \mathrm{~s}$

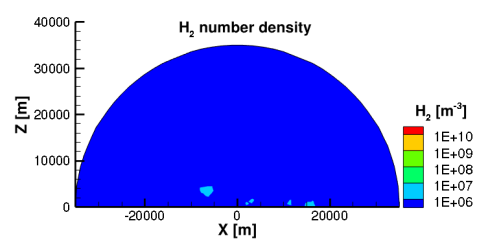

(j) $\mathrm{H}_{2}$ number density, $33.0 \mathrm{~s}$

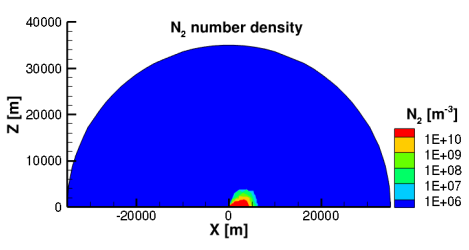

(b) $\mathrm{N}_{2}$ number density, $2.0 \mathrm{~s}$

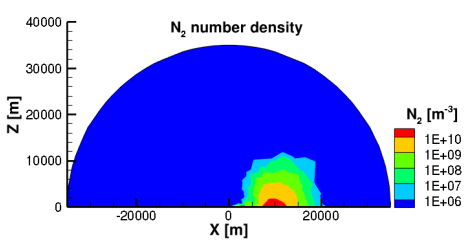

(e) $\mathrm{N}_{2}$ number density, $6.5 \mathrm{~s}$

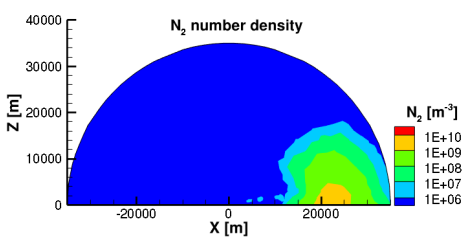

(h) $\mathrm{N}_{2}$ number density, $12.5 \mathrm{~s}$

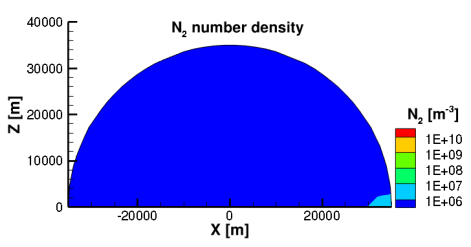

(k) $\mathrm{N}_{2}$ number density, $26.0 \mathrm{~s}$

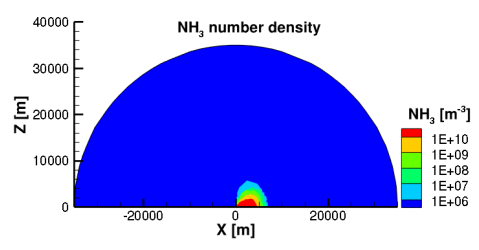

(c) $\mathrm{NH}_{3}$ number density, $2.0 \mathrm{~s}$

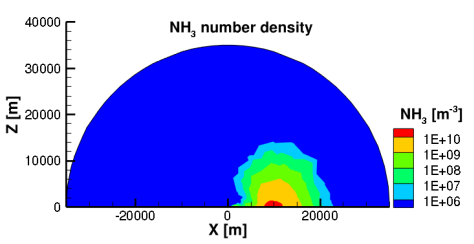

(f) $\mathrm{NH}_{3}$ number density, $6.5 \mathrm{~s}$

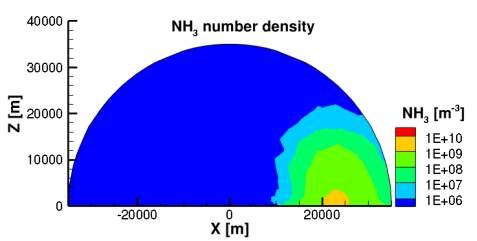

(i) $\mathrm{NH}_{3}$ number density, $12.5 \mathrm{~s}$

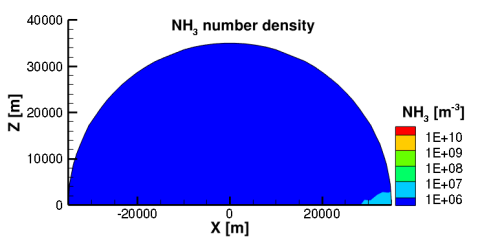

(l) $\mathrm{NH}_{3}$ number density, $28.5 \mathrm{~s}$

Figure 11. Time evolution of $\mathrm{H}_{2}, \mathrm{~N}_{2}$ and $\mathrm{NH}_{3}$ neutral plumes during an unsteady thruster firing with $\mathrm{B}=1.1 \times$ $10^{-7}$ T. Thrusters are fired for 2.0 seconds (top row) and then turned off. The lightest of the species, $\mathbf{H}_{2}$ exhibits the greatest plume diffusion, while the heaviest of the species, $\mathrm{NH}_{3}$ convects out of the computational domain first. 
tromagnetic field as observed from the number density contours. In previous work, ${ }^{7}$ the neutral plume was influenced indirectly by the magnetic field as a result of subsequent charge exchange from the ion plume chemical species back to neutral particles. This influence is not observed here, as the collisionality is considerably lower in the conditions examined at GEO. The electromagnetic field was found to influence the development of the ion plume. In the absence of the magnetic field, the electrostatic forces caused the spacecraft ions to accelerate outward from the thruster in $\pm x$-directions. When the magnetic field was introduced, the ion plumes developed a conical structure in the radial direction in the presence of the magnetic field. This may be attributed to the competition of the electrostatic acceleration and the confining gyroscopic motion by the magnetic field.

The unsteady simulation of the two-second thruster burn examined the relative neutral plume convection and dissipation at large distances $( \pm 35 \mathrm{~km})$ from the spacecraft. Although the corresponding ion plume is confined near the thruster origin, acting as a potential 'source' for neutral particle generation through charge exchange, this has effectively no influence on the dissipation of the neutral plumes. The neutral densities drop to values below the ambient, with only a few $\left(\mathbf{O}\left(10^{7}\right) \mathrm{m}^{-3}\right) \mathrm{H}_{2}$ neutral particles forming near the thruster origin after 33 seconds.

\section{Acknowledgments}

The support of Air Force Research Laboratory (AFRL) Space Vehicles Directorate and Barron Associates, Inc. for this work under subcontract FA9453-11-C-0181 is gratefully acknowledged. Computational resources and technical support were provided by the CAEN Advanced Computing Center at the University of Michigan, and by the Texas Advanced Computing Center through a National Science Foundation XSEDE award.

\section{References}

${ }^{1}$ Burke, W., Gentile, L., Machuzak, J., Hardy, D., and Hunton, D., "Energy distributions of thruster pickup ions detected by the Shuttle Potential and Return Electron Experiments during TSS 1," Journal of Geophysical Research, 100 A10, 1995.

${ }^{2}$ McMahon, W., Salter, R., Hills, R., and Delorey, D., "Measured electron contribution to shuttle plasma environment," AIAA-83-2598 AIAA Shuttle Environment and Operations Meeting, 1983.

${ }^{3}$ Karabadzhak, G., Plastinin, Y., Khmelinin, B., Teslenko, V., Shvets, N., Drakes, J., Swann, D., and McGregor, W., "Experimentation Using the Mir Station as a Space Laboratory," AIAA Aerospace Sciences Meeting, 1997, pp. AIAA-97-0288.

${ }^{4}$ Drakes, J. and Swann, D., "DSMC Computations of the Progress-M Spacecraft Retrofiring Exhaust Plume," AIAA Aerospace Sciences Meeting, January Reno, NV 1999, pp. AIAA-99-0975.

${ }^{5}$ Kaplan, C. and Bernhardt, P., "Effect of an Altitude-Dependent Background Atmosphere on Shuttle Plumes," Journal of Spacecraft and Rockets, Vol. 47, July-August 2010.

${ }^{6}$ Bernhardt, P., Ballenthin, J., Baumgardner, J., Bhatt, A., Boyd, I., Burt, J., Caton, R., Coster, A., Erickson, P., Huba, J., Earle, G., Kaplan, C., Foster, J., Groves, K., Haaser, R., Hellis, R., Hunton, D., Hysell, D., Klenzing, J., Larsen, M., Lind, F., Pedersen, T., Pfaff, R., Stoneback, R., Roddy, P., Rodriguez, S., San Antonio, G., Schuck, P., Siefring, C., Selcher, C., Smith, S., Talaat, E.R. an Thomason, J., Tsunoda, R., and Varney, R., "Ground and Space-Based Measurement of Rocket Engine Burns in the Ionosphere," IEEE Transactions on Plasma Science, Vol 40, No 5, 2012.

${ }^{7}$ Stephani, K. and Boyd, I., "Detailed modeling and analysis of spacecraft plume/ionosphere interactions in low Earth orbit," J. Geophys. Res. Space Physics, Vol. 119, 2014, pp. 2101-2116.

${ }^{8}$ Stephani, K., Boyd, I., Balthazor, R., McHarg, M., Mueller, B., and Adams, R., "Analysis and observation of spacecraft plume/ionosphere interactions during maneuvers of the space shuttle," J. Geophys. Res. Space Physics, Vol. 119, 2014, pp. 76367648.

${ }^{9}$ Bird, G., Molecular Gas Dynamics and the Direct Simulation of Gas Flows, Oxford University Press, Oxford, 1994.

${ }^{10}$ Birdsall, C. and Langdon, A., Plasma Physics via Computer Simulation, Taylor and Francis, New York, 2004.

${ }^{11}$ Cai, C., Theoretical and Numerical Studies of Plume Flows in Vacuum Chambers, Ph.D. Dissertation, University of Michigan, Ann Arbor, MI, 2005.

${ }^{12}$ Kusakabe, T., Kensuke, A., Gu, J., Hirsch, G., Buenker, R., Kimura, M., Tawara, J., and Nakai, Y., "Charge-transfer processes in collisions of $\mathrm{H}+$ ions with $\mathrm{H} 2, \mathrm{D} 2, \mathrm{CO}$ and $\mathrm{CO} 2$ molecules in the energy range 0.2-4.0 keV," Physical Review A, Vol. 62, 062714, 2000.

${ }^{13}$ Cabrera-Trujillo, R., Ohrn, Y., Deumens, E., Sabin, J., and Lindsay, B., "Theoretical and experimental studies of the $\mathrm{H}+-\mathrm{N} 2$ system: Differential cross sections for direct and charge-transfer scattering at kil-electron-volt energies," Physical Review A, Vol. 66, 042712, 2002.

${ }^{14}$ Coplan, M. and Ogilvie, K., "Charge Exchange for $\mathrm{H}+$ and $\mathrm{H} 2+$ in $\mathrm{H} 20, \mathrm{CO} 2$, and NH3," Journal of Chemical Physics, Vol. 52, 1970, pp. 4154.

${ }^{15}$ Rufenach, C., McPherron, R., and Schaper, J., "The Quiet Geomagntic Field at Geosynchronous Orbit and Its Dependence on Solar Wing Dynamic Pressure," J. Geophys. Res., Vol. 97, No. A1, 1992, pp. 25-42. 